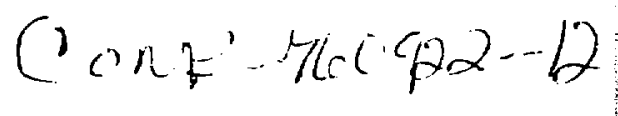

\section{Line Shape of Magnetic Excitations in} \\ Sing1et-Ground-State Systems* \\ Per Bak ${ }^{\dagger}$ \\ Brookhaven National Laboratory, Upton, NY 11973 \\ The excitation spectrum in a paramagnetic singlet \\ doublet system is calculated using a diagramatic expansion \\ technique, and the theoretical predictions are compared with \\ experiments on praseodymium. The theory gives an accurate \\ description of the dramatic temperature dependence of the \\ energies and lineshapes for the exciton modes.
}

For the last several years there hes been a great interest in the properties of localized magnetic systems which possess a nonmagnetic singlet ground state. The ordering in such systems occurs as an exchange polarization of the ground state, provided the exchange interaction between the magnetic ions exceeds a certain threshold value. Here we shall present a calculation of the paramagnetic excitation spectrum in a singlet doublet system using a diagramatic Green's function expansion method. A similar technique has been applied to a ferromagnet by Vaks, Larkin and Pikin $[1]$, and to the Ising model in a transverse field by

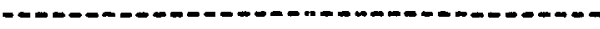

* Work supported by Energy Research and Development Administration.

$+\quad$ Present Address: NORDITA, Blegdamsvej 17, 2100 Copenhagen $\emptyset$, Denmark. CONTKACT NC. E(30-i) 16 
Stinchcombe [2]. In contrast to other techniques, based ou various decouplings of equations of motion for spin operators, this kind of theory gives a well defined hîh-density expansion parameter $(1 / z)$ where 2 If an effective number of interacting neighbors. The approximations are based on physical, not technical reasons. Praseodymium is an example of a singlet ground state magnet in which the exchange is only slightly undercritical with respect to magnetic ordering. The ground state on the hexagonal sites is the pure $\left[J^{2}=0>\right.$ singlet, and the first excited state is the doublet $|x\rangle,|y\rangle$, where $|x\rangle=1 / \sqrt{2}(|1\rangle+\mid-1>)$ and $\mid y>=-1 / \sqrt{2}(|1>-|-1>)$. The paramagnetic excitation spectrum has recently been measured by Houmann et al. [3] using inelastic neutron scattering technique. The lowest lying wode shows a clear tendency towards softening as the temperature is lowered toeards $0 \mathrm{~K}$, and a dramarfic increase of the intrinsic linewidth takes place as the temperature increases from $6 \mathrm{~K}$ to $30 \mathrm{~K}$, where.well-defined modes cease to exist. dhcp Praseodymium seems to be the simplest real singlet-ground-state magnet, and ignificantly more information is now available on the excitations In this material than in any other paramagnetic system. This makes the element $\operatorname{Pr}$ almost ideal for a confrontation between experiment and theoretical model calculations.

The effective Hamiltonian describing the magnetic ions on the hexagonal sites in $\operatorname{Pr}$ may be written

$$
\mathcal{A}=\sum_{1} \Delta\left(s_{i}{ }^{2}\right)^{2}-\sum_{1 j, \alpha \beta} \oint_{i j}^{\alpha \beta} s_{i}^{\alpha} s_{j}^{\beta}, \alpha, \beta=x, y
$$

$\Delta$ is the crystal field splitting $(=3.2 \mathrm{MeV})$. We introduce the Greon's functions 


$$
G^{\alpha \beta}\left(r_{1}, \tau_{1} ; r_{2}, \tau_{2}\right)=\left\langle\tau_{7} s^{\alpha}\left(r_{1}, \tau_{1}\right) s^{\beta}\left(r_{2}, \tau_{2}\right)\right\rangle
$$

where $\left\langle T_{7} \ldots\right\rangle$ denotes the thermal average of the $T$ ordered product of operators in the interaction representation. The Green's function can be represented by a sum of connected diagrams consistirg of single-

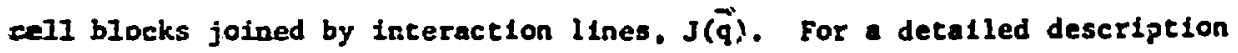
of the technique, see Ref. [4]. The zeroth order diagrans (RPA) simply sonsist of nonteteracting Green's function connected with interaction Iines. The poles of the RPA-propagators, wh1ch give the energy spectrum $\operatorname{are}$

$$
\left(\omega^{N}\right)^{2}-\Delta^{2}-4 \Delta R \oint^{N}(\bar{q})
$$

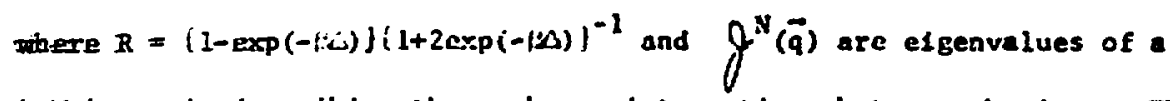
$4 \times 4$ matrix describing the exchange Interactions between the fons. The measured ifspersion relations wire stted to this expression and interatomic exchange paramecers wero Jerlved. Flgura I shows the polarizacion of two of the four mudes as a function of $q$ in thi $r-K-M d i r e c t i o n$. The polarizations chanpe rapidiy an a function of $q$ : at the zone center the polarizat on is IInear in the $x$ or $y$ direction, but in general the modes are elltpteally polarlacd in she xy plane. The elgenvectors of the modee can be measured by neut con ecetertnk teclingque.

The ligat order dlantase aloe rice to sall ohlfte of the exci-

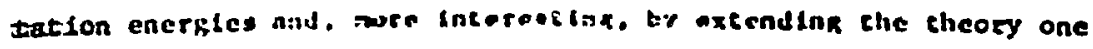
order beyond tho lipk ve o.tala the losting concributione to the llfectres

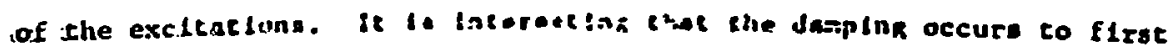

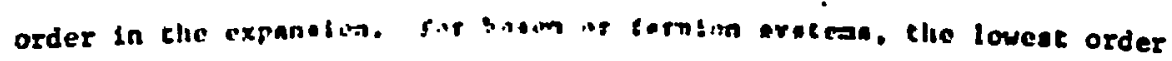

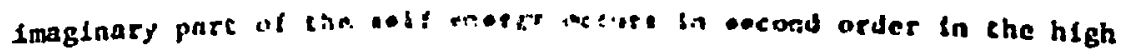


densfey expansion due to incaraction between excicacions. The spectral functions, which are proportional to the neturon scateering cross-section, can casily be expressed in terms of the Green's functions using the fluctuation-dissipation theores. The theoretical lineshapes convoluted with the experisental resolution function are coopared with experiment in Fig. 2. Almost complete agreement between positions of pesks, intensitles and Itneshapes is observed at any temperature. Tho agreenent is least perfect at the highese terpezatures, where the linewidth, as calculated to first order in the $\left(\frac{1}{2}\right)$ expansion, is somparable to the enerby as alculated to zeroth order. In addition to the diagrass considered here, there are also diagraes which, co first order in $\frac{1}{2}$, describes quasielastic contributions to $s(\vec{q}, \omega)$. Recently, a weak central peak has been observed in Pr at low cesperatures [6]. It would be interesting to calculate this effect within the present formisso and comare with experiment. 


\section{REEERENCES}

[1] V.G. Vaks, A.I. Larkin, and S.A. Pikin, Sov. Phys. JEIP 26 (1968) $188,647$.

[2] R.B. Seincheombe, J. riys. C6 (1973) 2484 .

(3) J.C. Houmann, M. Cluppelier, A.R. MackIntosh, P. Eak, O.D. MaMasters, and K.A. Gschneidner, Jr., Phys. Rev. Lete. 34 (1975) 587.

(4) Per Bak, Phys. Rev. Lett. 34 (2975) 1230; Phys. Hev. B12, (1975) 5203.

[5] J.G. Hourann and A.R. Macklntosh, prlvace comannlestion.

[6] J.C. Hounann, B. Lebech, A.R. Macklatosh, W.J.L. Buyers, O.D. Makasters and K.A. Gschneldnez, JT., this conference.

\section{FIGURE CAPTIONS}

F18. 1 Polertication of exciton modes In praseodyaim as a function of wavevector, $q$, In the not direction.

F18. 2 Spectral tunctions for menetic excitations in Pr. Polnts: Neution sessurements (Ref. [S\}); Lines: theoretlcal curves folded wich experimental resolution. 


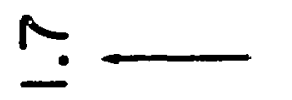

오
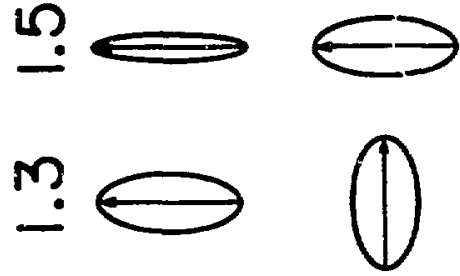

$\sim$

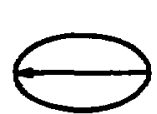

(1)

$=0$

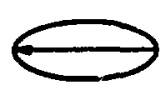

ㅇ.

๑. (1)

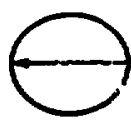

$\infty$

(1)

0

$m=$

0

$0 \quad 3$

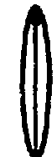

i
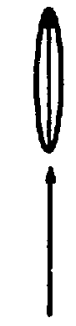

흐 


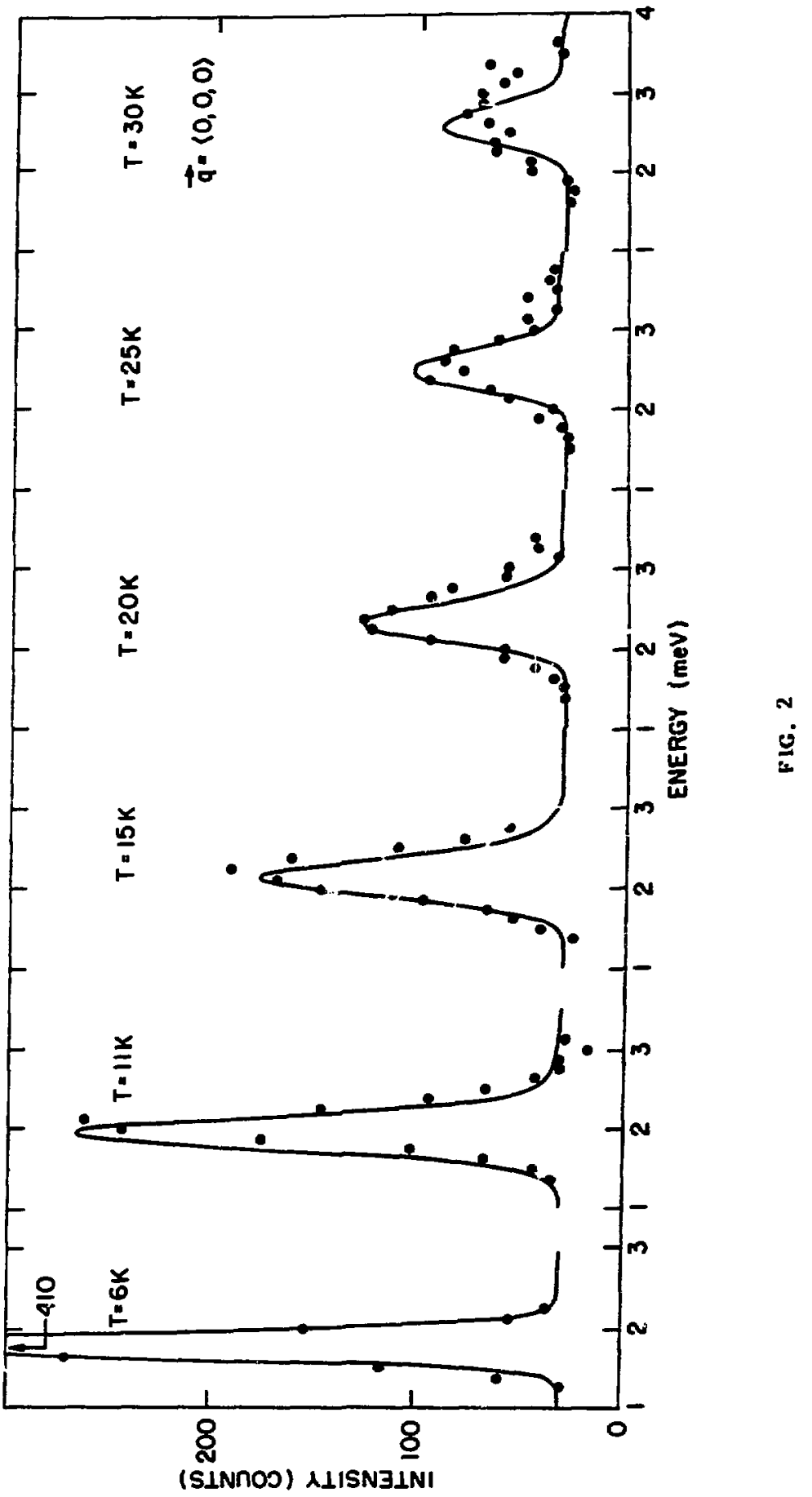

